\title{
Le Livre dit. Entretiens de "Duras filme », éd. Joëlle Pagès-Pindon, Paris, Gallimard, coll. « Les cahiers de la NRF », 2014, 240 p.
}

\section{Sandrine Vaudrey-Luigi}

\section{(2) OpenEdition}

\section{Journals}

Édition électronique

URL : https://journals.openedition.org/genesis/1609

DOI : 10.4000/genesis. 1609

ISSN : 2268-1590

Éditeur :

Presses universitaires de Paris Sorbonne (PUPS), Société internationale de génétique artistique littéraire et scientifique (SIGALES)

\section{Édition imprimée}

Date de publication : 9 mai 2017

Pagination : 224-226

ISBN : 979-1023-105636

ISSN : $1167-5101$

\section{Référence électronique}

Sandrine Vaudrey-Luigi, «Le Livre dit. Entretiens de " Duras filme », éd. Joëlle Pagès-Pindon, Paris, Gallimard, coll. "Les cahiers de la NRF », 2014, 240 p. », Genesis [En ligne], 44 | 2017, mis en ligne le 05 juin 2017, consulté le 16 mars 2023. URL : http://journals.openedition.org/genesis/1609 ; DOI : https://doi.org/10.4000/genesis. 1609 
auteur du Quichotte» de Jorge Luis Borges, Chartier achève sur des lignes qui évoquent «l'impossible rêve d'une œuvre toujours identique à elle-même» (p. 299). À l'illusion d'un âge d'or où l'écrit littéraire aurait connu un statut et une légitimité incontestables aujourd'hui mis à mal -, Chartier substitue la vision d'une ère moderne foncièrement complexe et dynamique.

Intitulée «Textes sans frontières» (chapitre IV), l'étude jusque-là inédite illustre bien ce parti qui attribue au texte un destin et des vies. Chartier y analyse en détail les traductions et éditions successives de la Brevísima relación de la destruyción de las Indias de Bartolomé de Las Casas (1552), ouvrage qui a joué un rôle de premier plan dans la propagation de la «légende noire» anti-espagnole. D'abord imprimé à Séville, le texte date de dix ans plus tôt, et une copie manuscrite en a été conservée. De retour d'Amérique, Las Casas dénonce dans ces lignes les atrocités de la colonisation telle qu'elle est organisée depuis les lois de Burgos de 1513. Dans un «Argument du présent sommaire» de l'édition de 1552, Las Casas précise que sa «relation» a été d'abord orale, et que la mise par écrit devait servir à alerter le roi Très Catholique de la situation dans ses colonies américaines. Chartier indique que le document a dû faire effet, puisque des réformes sont ordonnées à la fin de l'année 1542 déjà. Pourquoi alors le faire imprimer? Parce qu'en 1552, Las Casas est déçu de l'évolution des choses, et qu'il veut en appeler une nouvelle fois au roi, sous une forme «que Son Altesse [...] pût lire plus aisément» (p. 115). Pour donner plus de poids à son argumentaire, Las Casas fait imprimer en tout huit traités - dont la Brevísima relación - qui sont publiés sous une seule et même reliure. Chartier montre qu'ensuite, les trois traductions du texte publiées en terres protestantes (aux Pays-Bas espagnols et en Angleterre, entre 1578 et 1583) obéissent à des intentions de dénonciation politique, dans le contexte de la lutte des Pays-Bas contre leur souverain catholique, et dans celui de la rivalité anglo-espagnole de la fin du XVIe siècle. Autre usage et nouvelle interprétation, dans l'édition du texte parue à Francfort en 1598, dans le contexte des guerres religieuses : les cruautés espagnoles illustrées par Théodore de Bry servent à montrer ce dont les catholiques sont capables de commettre envers les peuples et les nations ayant adopté la Réforme. À Venise en 1626, à Barcelone en 1646 puis à Londres en 1656, de nouvelles éditions font écho aux préoccupations et aux intérêts politiques contemporains. Puis, à la fin du XVIIe siècle, le texte ressurgit dans plusieurs éditions en français, publiées à Paris, Amsterdam et Londres, qui présentent curieusement la Brevísima relación comme un «intéressant récit de voyage» (p. 136), à ranger aux côtés d'autres souvenirs pittoresques. Les descriptions des atrocités sont adoucies, quoique bien présentes, et des avertissements tentent d'en amoindrir la portée. Dernier avatar enfin, le texte paraît à nouveau en espagnol, à plusieurs reprises, dans les années 1810-1820 où les nations d'Amérique du Sud se soulèvent contre leurs maîtres européens.

Chartier clôt cet inventaire éclairant en remarquant: «La signification d'un texte ne dépend pas seulement de sa lettre. Elle est toujours construite par un double contexte : celui, historique, défini par les attentes de ses lecteurs, celui, matériel, donné par la présence dans le livre qui le publie de textes qui ne sont pas l'œuvre elle-même » (p. 143). En proposant d'associer histoire culturelle et critique textuelle, La Main de l'auteur et l'esprit de l'imprimeur offre d'innombrables pistes invitant à redécouvrir des œuvres de la tradition qui peuvent sembler figées dans leur interprétation.

Le Livre dit. Entretiens de «Duras filme », éd. Joëlle Pagès-Pindon, Paris, Gallimard, coll. «Les cahiers de la $\mathrm{NRF} », 2014,240 \mathrm{p}$.

\section{Compte rendu par Sandrine Vaudrey-Luigi*}

Tout commence avec la pièce Agatha, publiée le 3 mars 1981 aux Éditions de Minuit. Marguerite Duras entreprend aussitôt d'adapter son texte au cinéma avec une équipe relativement réduite. Dans le même temps, les proches qui l'entourent, Yann Lémée devenu très vite Yann Andréa - entré dans sa vie l'année précédente - son fils Jean Mascolo, surnommé Outa, et un ami, Jerôme Beaujour, la filment en train de réaliser son adaptation d'Agatha en vue de faire eux-mêmes un film intitulé Duras filme, documentaire qui paraît conjointement au film Agatha et les lectures illimitées, en octobre $1981 \ldots$ Le ton est donné : le volume édité par Joëlle Pagès-Pindon est le reflet d'une stratification et d'une mise en abyme jouant sur l'écrit, l'image, l'oral.

Le Livre dit. Entretiens de «Duras filme» est un volume provenant d'archives personnelles de Jean Mascolo, que ce dernier a confié à une des spécialistes de Marguerite Duras, Joëlle Pagès-Pindon. Ces archives consistaient en trois cassettes audiovisuelles contenant environ six heures d'enregistrement, plus précisément de rushes, c'est-à-dire de l'ensemble des prises de vue filmées en vue du montage de Duras filme: la première partie du volume, «Les entretiens de Duras filme», en propose une transcription. Mais à cette première strate s'en ajoute une autre puisque la seconde partie du volume est issue d'un dossier d'archives portant la mention «écrits à déchiffrer», composé de feuillets dactylographiés transcrivant des passages de Duras filme: il s'agissait pour J. Pagès-Pindon d'établir un texte à partir d'une transcription dactylographiée de Duras filme, transcription abondamment revue par Duras...

Précédant ces deux sections, la «Préface» et la «Note sur l'édition du texte» apportent plusieurs formes d'informations. La première partie de la préface contextualise les deux sections du volume en mettant en évidence l'importance du livre de chroniques L'Été 80 ainsi que celle de l'entrée dans la vie de l'écrivain de Yann Andréa - «vecteur de son imaginaire et [...] centre scriptural de sa création» (J. PagèsPindon, p. 17) - à la fin de l'été 1980. C'est également l'occasion de souligner à quel

(*) Université Sorbonne Nouvelle-Paris 3, Clesthia. 
point le tournage d'Agatha et les lectures illimitées a réactivé chez Duras des souvenirs liés au «petit frère» tant aimé. La seconde partie de la préface s'arrête plus précisément sur les conditions de réalisation des Entretiens de Duras filme, sur l'intérêt selon l'éditrice de publier la transcription de ces rushes, dans la mesure où Duras « contribue à nous rendre visible la cohérence de sa création » (p. 25). Quant aux enjeux de la seconde section du volume, ils sont encore plus évidents puisque ces feuillets étaient jusqu'alors restés inédits. La «Note sur l'édition du texte» permet classiquement à J. Pagès-Pindon de s'expliquer sur le travail qu'elle a accompli pour transcrire d'une part les cassettes audiovisuelles, d'autre part pour établir un texte à partir du tapuscrit remanié par Duras de Duras filme, établissement dont la difficulté est bien rendue par la reproduction du folio $\mathrm{n}^{\circ} 7$ (p. 183). De fait comme le souligne J. Pagès-Pindon (p. 30), «Les vingt-huit feuillets qui composent le manuscrit des "Brouillons $d u$ Livre dit" ont des allures de palimpseste».

L'intérêt de ce volume dépasse largement celui de présenter des inédits tant ils s'inscrivent dans la production durassienne d'une manière plus globale. Tout d'abord, ils éclairent d'un nouveau jour la période des années 1980-1982 : alors que les chroniques de L'Été 80, Agatha ou encore La Maladie de la mort véhiculent une représentation de Marguerite Duras relativement seule, notamment face à la rencontre fulgurante avec Yann Andréa puis au désenchantement qui a suivi, le lecteur la découvre entourée de ses proches, aimant non seulement partager des moments avec eux, échanger des idées, mais également appréciant répondre à leurs sollicitations, notamment à celles de Yann Andréa, ou prenant simplement du plaisir à parler :

Vous savez, moi, vous me mettez devant une caméra, je peux parler pendant huit heures! Je ne l'ai jamais fait, mais je peux le faire.

Yann Andréa - Sur quoi par exemple? M. D. - Tout, tout, absolument sur tout (p. 42).
De fait, Duras aborde une grande variété de sujets pendant qu'elle est filmée filmant Agatha ou les lectures illimitées. J. PagèsPindon a intitulé les principales séquences afin de guider le lecteur; ces dernières fournissent un résumé fidèle de l'ensemble du contenu : «Le désir, l'inceste, l'homosexualité», «Filmer Agatha et La jeune fille et l'enfant à Trouville», «Les robes de Bulle Ogier», «En tournage avec Bulle Ogier», «Les bonheurs de l'été 80 », «Prises de vue : la mer, les arbres», «Les livres et les films, la mort, les femmes et les hommes », «En tournage avec Yann Andréa», «Le cinéma, le "gai désespoir", l'amour maternel ». Ces titres rendent compte également de l'alternance entre des sujets sérieux, graves, et la légèreté qui peut accompagner certains moments du tournage; en ce sens, c'est également toute la richesse d'une ambiance qui nous est restituée, et c'est certainement l'un des plus grands intérêts de ce volume.

Ensuite, ces inédits rappellent une fois de plus la porosité qui existe dans l'œuvre durassienne non seulement entre l'écrit et l'oral, mais également entre l'écriture et le cinéma. Ainsi ce volume est-il une formidable illustration de ce que Duras a déclaré dans Les Yeux verts, recueil de textes publiés en juin 1980 dans Les Cahiers du cinéma («Je parle aussi de l'écrit même quand j'ai l'air de parler du cinéma. Je ne sais pas parler d'autre chose. Quand je fais du cinéma, j'écris ${ }^{1}$ »), ou encore précisément à l'époque d'Agatha ( «Je voudrais parler de l'écrit et de ma voix. Ma voix, tu dois l'entendre quand tu $\operatorname{lis}^{2} \gg$ ), mais également plus tard, lorsqu'elle livre ses textes testamentaires («Écrire, c'est très près du rythme de la parole $\left.{ }^{3} »\right)$. Or cette porosité a une conséquence fondamentale : les grandes constantes de l'idiolecte durassien sont également observables dans ce volume, notamment dans la seconde section. Déjà présente dans la première section («l'infini de la mer et l'infini de l'enfant», p. 65), on observe notamment cette configuration de la substantivation de l'adjectif avec inversion du caractérisé et du caractérisant, qui en tant que signal de la langue littéraire en général baroquise la prose chez
Duras : «cet appauvrissement nouveau du sentiment» (p. 185); « l'immensité de cette relation» (p. 191); « la crapulerie essentielle de l'État soviétique» (p. 193-194).

Enfin, la seconde section du volume présente un double intérêt spécifique : d'une part, ces feuillets dactylographiés largement corrigés, biffés et annotés par Duras permettent non seulement de mesurer ce que la retranscription initiale a conservé de l'ensemble des heures de tournage pour Duras filme, mais surtout ils offrent un exemple du travail de l'auteur à partir d'une version initiale. Et si J. Pagès-Pindon utilise le terme de «palimpseste», c'est que le travail d'établissement du texte est souvent rendu difficile par les multiples campagnes de corrections voire de réécritures menées sur un même feuillet, sans qu'elles soient toutes précisément datables - ainsi J. Pagès-Pindon réussit-elle à situer l'une d'entre elles par une allusion de Duras à l'attentat de la rue des Rosiers. L'autre intérêt manifeste de cette seconde section du volume réside, pourrait-on dire, dans le gain stylistique obtenu à partir des séquences de tournage de Duras filme. Dans la mesure où ces feuillets étaient destinés à une publication - même si cette dernière n'a jamais vu le jour-les paroles se trouvent condensées, les formules retravaillées deviennent plus lapidaires, les jugements se font aussi souvent plus sentencieux, comme ces propos sur l'homosexualité :

L'homosexualité n'existe pas sans l'hétérosexualité. L'hétérosexualité existe sans modèle, seule. L'homosexualité est une façon de remplacement de l'hétérosexualité, c'en est une parodie affaiblie et un plagiat aussi très codé, faute d'avoir la force d'affronter la tragédie de l'hétérosexualité (p. 190).

1. M. Duras, Les Yeux verts, Paris, Les Cahiers du cinéma, coll. "Petite Bibliothèque des Cahiers du cinéma», 1996, p. 76.

2. Marguerite Duras à Montréal, dir. S. Lamy et A. Roy, Montréal, Spirale-Solin, 1984, p. 57. 3. M. Duras, C'est tout, Paris, P.O.L, 1995, p. 15 . 
Mais ces feuillets laissent également affleurer des propos plus légers, ancrés dans le temps du tournage et qui pourtant laissent percevoir par exemple l' aplomb de l'écrivain qui amuse ses proches :

Nous allons tourner très peu, vingt plans (rire général), vingt-cinq plans et puis nous savons déjà que le film fera le tour de l'intelligence du cinéma (p. 192).

C'est parce que je ne sais pas jouer que ma musique est tellement belle (p. 192).

Ce volume est donc plus qu'un document sur la vie quotidienne d'un tournage. Il est tout d'abord, un complément à l'édition d'Agatha dans la collection de la «Bibliothèque de la Pléiade», texte édité également par J. Pagès-Pindon sous la direction de Gilles Philippe et paru en 2014. Il constitue une somme passionnante pour le cinéphile qui regarde un film en train de se faire, pour le généticien découvrant le travail de l'écrivain sur des brouillons inédits, pour tout spécialiste ou tout lecteur de Duras qui peut une fois de plus mesurer la proximité de l'oral et de l'écrit chez Marguerite Duras, ou à quel point l'écrit s'inscrit dans l'oral tout en s'affranchissant.

Hannah Sullivan, The Work of Revision, Cambridge, Harvard University Press, 2013, 360 p.

\section{Compte rendu par Alienor Vauthey*}

Dans The Work of Revision, c'est d'abord une histoire que nous raconte Hannah Sullivan : celle de la révision chez les Anglo-Saxons, du XIXe siècle à nos jours. Dans cet intervalle, la révision, d'abord exercée dans la douleur et le secret, deviendrait un gage essentiel de la valeur littéraire du texte, selon une évolution qui prendrait ses racines dans le mouvement moderniste et la culture de l'imprimerie. Par ailleurs, à travers l'analyse de manuscrits, tapuscrits, épreuves et propos métalittéraires de plusieurs grands écrivains modernes anglo-saxons, Sullivan se propose de répondre à la question suivante : «Comment les aspects thématiques et formels d'un texte sont-ils liés à sa genèse ?» (p. 5).

L'auteur de l'étude appuie son travail sur plusieurs écoles de pensée telles que la critique génétique française (on trouve en bibliographie les noms de Pierre-Marc de Biasi, Daniel Ferrer et Louis Hay) et le social-text editing; elle en tire une méthode d'investigation «plus pratique que théorique» (p. 60), qui lui permet de se concentrer sur le geste auctorial sans toutefois négliger les intentions d'autres acteurs ayant pu prendre part à l'élaboration du texte d'une manière ou d'une autre. Le cas le plus marquant est sans aucun doute celui d'Eliot, que les conseils d'Ezra Pound ont amené à supprimer une grande partie de son célèbre poème The Waste Land. Arguant de la grande dispersion des institutions de conservation anglo-saxonnes, Sullivan précise qu'elle a parfois dû se fier à la littérature secondaire plutôt qu'à sa propre analyse des documents. Dans le contexte de ce numéro de Genesis, il importe de souligner que sous le terme de «revision» l'auteur envisage les réécritures aussi bien avant qu' après édition, des ratures du brouillon à celles qu'on peut observer dans les marges d'un livre publié. Plus tard dans l'ouvrage (chap. II), à propos de Dencombe, l'écrivain mis en scène par James dans «The Middle Years», Sullivan propose une passionnante réflexion sur la révision post-éditoriale. Elle oppose alors relecture (rereading), qui altère l'interprétation sans modifier la lettre, et revision, qui destabilise la lettre, parfois dans le but même de refixer le sens. Elle observe alors : «La révision postéditoriale promet à l'auteur la possibilité de se réapproprier le texte alors que celui-ci a déjà commencé à circuler dans la sphère publique et, par conséquent, de refixer sa signification à son point d'origine» (p. 78). Mais elle constate que le désir de réancrer le sens dans l'intention de l'auteur qui motiverait (du moins chez cette figure d'écrivain qu'est Dencombe) la révision chronique conduit, paradoxalement, à une prolifération des versions qui affaiblit l'autorité du texte plutôt qu'elle ne la renforce.
L'histoire de la révision proposée par Sullivan commence avec les romantiques, dont le culte de l'écriture spontanée conduit à condamner par principe un processus qui remet en question l'inspiration première. En ce temps-là, qui plus est, la révision post-éditoriale est une pratique coûteuse. L'invention du linotype en 1886 , qui permet l'accélération de la composition des plaques d'imprimerie et facilite leur réagencement, atténue l'importance de cet obstacle. Le développement de la machine à écrire contribue par ailleurs à stimuler les processus de révision dans les stades prééditoriaux cette fois, puisque, selon Sullivan, «avoir la possibilité de voir les textes fixés sous de nombreuses formes différentes semble promouvoir la fluidité textuelle» (p. 8). À l'aube du XXe siècle, la tendance s'inverse ainsi progressivement : pour les modernistes, grands adeptes de l'expérimentation, les méthodes de révision se présentent comme de bons moyens d'explorer les possibilités d'un texte et de dépasser ce que l'on considère désormais comme la superficialité d'un premier jet. Mais les préjugés ont la peau dure, et la révision demeure encore une pratique seulement à demi avouable. Nombreux sont les auteurs de la première moitié du siècle, comme Woolf, James ou Eliot, qui la pratiquent sans vraiment le confesser. Ce n'est qu' après la guerre que les auteurs se mettent à afficher sans honte leur culte ; tandis que le modernisme institutionnalisé envahit écoles et universités, les processus d'écriture passent sous la loupe; les cours d'écriture créative se mettent à fleurir, qui contribuent à enseigner l'idée qu'il faut réviser. Le fameux slogan de Pound, «make it new », résonne dans les esprits et présente la révision comme un moyen de faire du neuf avec du vieux.

Cette évolution de la pratique de révision soulève une question qui court dans l'ensemble du livre : qu'est-ce que la «bonne» version d'un texte? Pour aborder cette question philologique essentielle,

(*) Université de Lausanne. 